\section{Stability Analysis for a Class of Partial Differential Equations via Semidefinite Programming}

\author{
Giorgio Valmorbida, Mohamadreza Ahmadi, \\ Antonis Papachristodoulou
}

\begin{abstract}
This paper studies scalar integral inequalities in one-dimensional bounded domains with polynomial integrands. We propose conditions to verify the integral inequalities in terms of differential matrix inequalities. These conditions allow for the verification of the inequalities in subspaces defined by boundary values of the dependent variables. The results are applied to solve integral inequalities arising from the Lyapunov stability analysis of partial differential equations. Examples illustrate the results.
\end{abstract}

Index Terms-Distributed Parameter Systems, PDEs, Stability Analysis, Sum of Squares.

\section{INTRODUCTION}

The need for accurate models to study dynamical systems [1], [2], [4], [30] have driven research efforts towards partial differential equation (PDE) systems - equations involving derivatives with respect to more than one independent variable. Such infinite dimensional system models are challenging to study both analytically and numerically. Conventional numerical approaches to study PDEs rely on spectral or spatial discretization and use tools developed for Ordinary Differential Equations (ODEs) [10], [7]. Numerical methods which do not require finite-dimensional approximations are needed may prevent conservatism in the system analysis.

Stability analysis of PDE systems can be performed with the Lyapunov's second method, which was extended to infinite dimensional systems in [6], [17]. One advantage of this method is that it does not require calculation of semi-groups [16], but instead requires the construction of a Lyapunov Functional (LF). A priori choices for LF structures for a given system are difficult to make. In particular cases, for instance when the PDE contains energy-preserving non-linearities such as convection terms, taking the energy of the state as the LF simplifies the stability analysis [25]. The Lyapunov conditions for PDE systems take the form of integral inequalities, therefore methods for computing the LFs require the solution of integral inequalities.

This paper presents a method to verify integral inequalities with integrands given by polynomials in the dependent variables. The polynomial structure allows for a quadraticlike representation of the integrand and we formulate conditions for the positivity of integral expressions in

The authors are with the Department of Engineering Science, University of Oxford, Parks Road, Oxford, OX1 3PJ, UK e-mail: (\{ giorgio.valmorbida, mohamadreza.ahmadi, antonis \}@eng.ox.ac.uk), tel +441865283035 . G. Valmorbida is also affiliated to Somerville College, University of Oxford, Oxford, U.K. Work supported by EPSRC grant EP/J010537/1. M. Ahmadi is supported by the Oxford Clarendon Scholarship and the Sloane-Robinson Scholarship. terms of differential matrix inequalities. The proposed method is then applied to study the stability of systems of inhomogeneous PDEs, using weighted $\mathcal{H}^{q}$ norms (on Hilbert spaces) as LF candidates.

For the case of integrands that are polynomial in the independent variables as well, the differential matrix inequality formulated by the proposed method becomes a polynomial matrix inequality. We then exploit the semidefinite programming (SDP) formulation [21] of optimization problems with linear objective functions and polynomial (sum-of-squares (SOS)) constraints [3] to obtain a numerical solution to the integral inequalities. Several analysis and feedback design problems have been studied using polynomial optimization: the stability of time-delay systems [23], synthesis control laws [24], [29], applied to optimal control law design [15] and system analysis [11]. In the context of PDEs, preliminary results have been presented in [19], where a stability test was formulated as a SOS programme (SOSP) and in [27] where quadratic integrands were studied.

The paper is organised as follows. Section II defines the problem, illustrates how we can write polynomial integrands in quadratic-like forms and proposes a method to verify integral inequalities by studying matrix inequalities associated with quadratic-like representation of the integrands. In Section III, some results for the stability analysis of PDE systems are summarised. Two examples illustrate the application of the proposed method to the stability analysis of PDEs in Section IV. Finally, Section V concludes the paper and gives directions for future research.

Notation. Let $\mathbb{R}, \mathbb{R}_{\geq 0}, \mathbb{R}_{>0}$ and $\mathbb{R}^{n}$ denote the field of reals, non-negative reals, positive reals and the $n$ dimensional Euclidean space, respectively. The sets of natural numbers and positive natural numbers are denoted $\mathbb{N}_{0}, \mathbb{N}$. The closure of set $\Omega$ is denoted $\bar{\Omega}$. The boundary $\partial \Omega$ of set $\Omega$ is defined as $\bar{\Omega} \backslash \Omega$ with $\backslash$ denoting set subtraction. The ring of polynomials, the ring of positive polynomials, and the ring of sum-of-squares polynomials on a real variable $x$ are denoted $\mathcal{R}[x], \mathcal{P}[x]$ and $\Sigma[x]$ respectively. The ring of SOS square matrices of dimension $n$, i.e., matrices $M(x) \in \mathcal{R}^{n \times n}[x]$ satisfying $M(x)=$ $\sum_{i=1}^{n_{M}} N_{i}^{T}(x) N_{i}(x)$ with $N_{i}(x) \in \mathbb{R}^{d_{i} \times n}$, is denoted $\Sigma^{n \times n}[x]$. For $n, k \in \mathbb{N}$, define the matrix $K \in \mathbb{N}^{n \times \sigma(n, k)}$, $\sigma(n, k):=\left(\begin{array}{c}n+k-1 \\ n-1\end{array}\right)=\frac{(n+k-1) !}{(n-1) ! k !}$, of which the columns satisfy $\sum_{i=1}^{n} K_{i j}=k, \forall j$, without repetition. The multiindex notation is used to define the vector of all monomials of degree $k \in \mathbb{N}$ on vector $w=\left(w_{1}, w_{2}, \ldots, w_{n}\right) \in \mathbb{R}^{n}$, as $w^{\{k\}}:=\left[\begin{array}{lll}\left(w^{K_{(\cdot 1)}}\right)^{T} & \cdots & \left(w^{K_{(\cdot \sigma(n, k)}}\right)^{T}\end{array}\right]$ where $w^{K_{(\cdot j)}}=$ $\prod_{i=1}^{n} w_{i}^{K_{i j}}$. The number of terms in $w^{\{k\}}$ is hence given by $\sigma(n, d)$. For instance, with $n=2$ and $k=2$ one has $K=\left[\begin{array}{lll}2 & 1 & 0 \\ 0 & 1 & 2\end{array}\right]$, and $w^{\{2\}}=\left(w_{1}^{2}, w_{1} w_{2}, w_{2}^{2}\right)$. Define the vector containing all monomials in $w$ up to degree $k$ as $\eta^{k}(w):=\left[\begin{array}{llll}1 & \left(w^{\{1\}}\right)^{T} & \ldots & \left(w^{\{k\}}\right)^{T}\end{array}\right]^{T}$. The set of continuous vector functions, mapping $\Omega \subset \mathbb{R}$ into $\mathbb{R}^{n}$, which are $k$-times differentiable and have continuous derivatives is denoted $\mathcal{C}^{k}(\Omega)$. For $p(x) \in \mathcal{C}^{1}(\Omega)$, the $d$ - 
th derivative of $p$ with respect to variable $x$ is denoted $\partial_{x}^{d} p$. For $u \in \mathcal{C}^{\alpha}(\Omega), \alpha \in \mathbb{N}_{0}$, define $D^{\alpha} u=v_{\alpha}(u):=$ $\left(u, \partial_{x} u, \ldots, \partial_{x}^{\alpha} u\right), \alpha$ is the order of $D^{\alpha} u$. The variable $u$ is called the dependent variable and $x \in \Omega$, the independent variable. For notational convenience, depending on the context we may suppress the dependence of $u(t, x)$ on $t$ (by writing $u(x)$ ) or $x$ (by writing $u(t)$ ) or both (by writing $u$ ). The set of functions satisfying $u \in \mathcal{C}^{\alpha}(\Omega)$ for which $\|u\|_{\alpha}^{2}=\int_{\Omega}\left(D^{\alpha} u(x)\right)^{T}\left(D^{\alpha} u(x)\right) \mathrm{d} x<\infty$ is denoted $\mathcal{H}^{\alpha}(\Omega)$. For a matrix $P(x)>0 \forall x \in \Omega$, define the norm $\|u\|_{(\alpha, P(x))}:=\left(\int_{\Omega}\left(D^{\alpha} u(x)\right)^{T} P(x)\left(D^{\alpha} u(x)\right) \mathrm{d} x\right)^{\frac{1}{2}}$. The set of real symmetric matrices is denoted $\mathbb{S}^{n}=\{A \in$ $\left.\mathbb{R}^{n \times n} \mid A=A^{T}\right\}$. For $A \in \mathbb{S}^{n}$, denote $A \geq 0(A>0)$ if $A$ is positive semidefinite (definite). The ceil function is denoted $\lceil\cdot 7$. The Jacobian of a vector field is denoted by $\nabla$, the Laplacian is denoted $\nabla^{2}$ and the cross-product is denoted by the symbol $\times$.

\section{INTEGRAL INEQUALITIES WITH POLYNOMIAL INTEGRANDS}

In this section, we study inequalities given by polynomials on the dependent variables evaluated at the boundaries of the domain of integration and integral terms with polynomial integrands on the dependent variables.

Consider the integral inequality

$$
f_{b}\left(v_{\theta-1}(u(1)), v_{\theta-1}(u(0))\right)+\int_{\Omega} f_{i}\left(x, v_{\theta}(u(x))\right) \mathrm{d} x \geq 0,
$$

with $\Omega=[0,1], f_{b} \in \mathcal{R}\left[v_{\theta-1}(u(1)), v_{\theta-1}(u(0))\right], f_{i}\left(\cdot, v_{\theta}\right) \in$ $\mathcal{R}\left[v_{\theta}\right]$ ( $f_{i}$ is a polynomial on its second argument). In order to simplify the exposition, let us define

$$
v_{\theta-1}^{b}(u):=\left[\begin{array}{ll}
\left(v_{\theta-1}(u(1))\right)^{T} & \left(v_{\theta-1}(u(0))\right)^{T}
\end{array}\right]^{T} .
$$

For $\max \left(\operatorname{deg}\left(f_{b}\right), \operatorname{deg}\left(f_{i}\right)\right)=k$, we can express the polynomials $f_{b}$ and $f_{i}$ as in the quadratic-like forms

$$
\begin{aligned}
& f_{b}\left(v_{\theta-1}^{b}\right)=\left(\eta^{\left\lceil\frac{k}{2}\right\rceil}\left(v_{\theta-1}^{b}(u)\right)\right)^{T} F_{b} \eta^{\left\lceil\frac{k}{2}\right\rceil}\left(v_{\theta-1}^{b}(u)\right) \\
& f_{i}\left(x, v_{\theta}(u)\right)=\left(\eta^{\left\lceil\frac{k}{2}\right\rceil}\left(v_{\theta}(u)\right)\right)^{T} F_{i}(x) \eta^{\left\lceil\frac{k}{2}\right\rceil}\left(v_{\theta}(u)\right)
\end{aligned}
$$

with symmetric matrix $F_{b} \in \mathbb{S}^{\sigma\left(2 n \theta,\left\lceil\frac{k}{2}\right\rceil\right) \times \sigma\left(2 n \theta,\left\lceil\frac{k}{2}\right\rceil\right)}$ and symmetric matrix function $F_{i}: \Omega \rightarrow \mathbb{S}^{\sigma\left(n \theta,\left\lceil\frac{k}{2}\right\rceil\right) \times \sigma\left(n \theta,\left\lceil\frac{k}{2}\right\rceil\right)}$. The dependent variable $u(x)$ is assumed to belong to sets of the form

$$
\mathcal{B}(B):=\left\{u \in \mathcal{C}^{\theta-1}(\Omega) \mid B v_{\theta-1}^{b}=0\right\},
$$

with $B \in \mathbb{R}^{n_{b} \times 2 n \theta}$, where $n_{b}$ is the number of constraints on the boundary.

We study the following problem

Problem 1. Check whether the integral inequality

$$
f_{b}\left(v_{\theta-1}^{b}(u)\right)+\int_{\Omega} f_{i}\left(x, v_{\theta}(u)\right) \mathrm{d} x \geq 0
$$

holds for all $u \in \mathcal{H}^{\theta}$ satisfying $u \in \mathcal{B}(B)$.
For a given polynomial $f_{b}$, the representation (1) may be non-unique and is taken as an element of the set

$$
\begin{aligned}
& \mathcal{F}_{b}(k, \theta)=\left\{F_{b}+G_{b}: \mathbb{S}^{\sigma\left(n \theta,\left\lceil\frac{k}{2}\right\rceil\right) \times \sigma\left(n \theta,\left\lceil\frac{k}{2}\right\rceil\right)}\right. \\
& \mid f_{i}=\left(\eta^{\left\lceil\frac{k}{2}\right\rceil}\left(v_{\theta-1}^{b}\right)\right)^{T} F_{b} \eta^{\left\lceil\frac{k}{2}\right\rceil}\left(v_{\theta-1}^{b}\right), \\
&\left.0=\left(\eta^{\left\lceil\frac{k}{2}\right\rceil}\left(v_{\theta-1}^{b}\right)\right)^{T} G_{b} \eta^{\left\lceil\frac{k}{2}\right\rceil}\left(v_{\theta-1}^{b}\right)\right\}
\end{aligned}
$$

Similarly, for a given function $f_{i}$, the set of quadratic-like representation (2) is taken as an element of the set

$$
\begin{gathered}
\mathcal{F}_{i}(k, \theta)=\left\{F_{i}(x)+G_{i}(x): \Omega \rightarrow \mathbb{S}^{\sigma\left(n \theta,\left\lceil\frac{k}{2}\right\rceil\right) \times \sigma\left(n \theta,\left\lceil\frac{k}{2}\right\rceil\right)}\right. \\
\mid f_{i}=\left(\eta^{\left\lceil\frac{k}{2}\right\rceil}\left(v_{\theta}(u)\right)\right)^{T} F_{i}(x) \eta^{\left\lceil\frac{k}{2}\right\rceil}\left(v_{\theta}(u)\right) \\
\left.0=\left(\eta^{\left\lceil\frac{k}{2}\right\rceil}\left(v_{\theta}(u)\right)\right)^{T} G_{i}(x) \eta^{\left\lceil\frac{k}{2}\right\rceil}\left(v_{\theta}(u)\right)\right\}
\end{gathered}
$$

The following example illustrates the above definitions.

Example 1. Consider $f_{i}\left(x,\left(u, \partial_{x} u\right)\right)=x^{2} \partial_{x} u+2 u\left(\partial_{x} u\right)^{2}$, $u \in\{u \mid u(0)=u(1)\}$, yielding $k=3, \theta=1$, and $v_{\theta}(u)=$ $\left(u, \partial_{x} u\right), \eta^{\left\lceil\frac{k}{2}\right\rceil}\left(v_{\theta}(u)\right)=\left(1, u, \partial_{x} u, u^{2}, u \partial_{x} u,\left(\partial_{x} u\right)^{2}\right)$. The quadratic representation (2) and the set (3) are obtained with $v_{\theta-1}^{b}=[u(1) u(0)]$,

$$
F_{i}(x)=\left[\begin{array}{cccccc}
0 & 0 & \frac{x^{2}}{2} & 0 & 0 & 0 \\
0 & 0 & 0 & 0 & 0 & 1 \\
\frac{x^{2}}{2} & 0 & 0 & 0 & 0 & 0 \\
0 & 0 & 0 & 0 & 0 & 0 \\
0 & 0 & 0 & 0 & 0 & 0 \\
0 & 1 & 0 & 0 & 0 & 0
\end{array}\right], B=\left[\begin{array}{ll}
1 & -1
\end{array}\right] .
$$

The set $\mathcal{F}_{i}(k, \theta)$ is defined by matrices $G_{i}(x)$ as

$$
G_{i}(x)=\left[\begin{array}{cccccc}
0 & 0 & 0 & g_{1}(x) & g_{2}(x) & g_{3}(x) \\
0 & -2 g_{1}(x) & -g_{2}(x) & 0 & 0 & 0 \\
0 & -g_{2}(x) & -2 g_{3}(x) & 0 & 0 & 0 \\
g_{1}(x) & 0 & 0 & 0 & 0 & g_{4}(x) \\
g_{2}(x) & 0 & 0 & 0 & -2 g_{4}(x) & 0 \\
g_{3}(x) & 0 & 0 & g_{4}(x) & 0 & 0
\end{array}\right] .
$$

However, a complete quadratic representation of the integral expression (4), must also account for the differential relation among the entries of $v_{\theta}(u)$ and is characterized by the set $\mathcal{I}$ as in (7). Notice that the set is obtained by a straightforward application of the fundamental theorem of calculus, which allows us to introduce matrices $H_{b}$ related to the values at the boundary and matrix functions $H_{i}(x) \in \mathcal{C}^{1}(\Omega)$.

The example below illustrates matrices $H_{b}$ and $H_{i}(x)$ for an element of set (7).

Example 2. Consider (4) with $f_{b}=2 u(1) \partial_{x} u(0)$, $f_{i}\left(x,\left(u, \partial_{x} u\right)\right)=\sin ^{2}(x) u \partial_{x} u+2 u \partial_{x}^{2} u$, yielding $k=2$, $\theta=2$, and $v_{\theta}(u)=\left(u, \partial_{x} u, \partial_{x}^{2} u\right)$. Since the expression is homogeneous of degree $k=2$, we replace the inhomogeneous vector $\eta^{\left\lceil\frac{k}{2}\right\rceil}\left(v_{\theta}(u)\right)$ by a homogeneous vector $\left(v_{\theta}(u)\right)^{\left\{\frac{k}{2}\right\}}$ to obtain the quadratic expressions with $\left(v_{\theta-1}^{b}\right)^{\{1\}}=\left(u(1), \partial_{x} u(1), u(0), \partial_{x} u(0)\right),\left(v_{\theta}(u)\right)^{\{1\}}=$ $\left(u, \partial_{x} u, \partial_{x}^{2} u\right)$. The representation (2) is defined by

$$
F_{b}=\left[\begin{array}{llll}
0 & 0 & 0 & 1 \\
0 & 0 & 0 & 0 \\
0 & 0 & 0 & 0 \\
1 & 0 & 0 & 0
\end{array}\right], F_{i}(x)=\left[\begin{array}{ccc}
0 & -\frac{\sin ^{2}(x)}{2} & 1 \\
-\frac{\sin ^{2}(x)}{1^{2}} & 0 & 0 \\
0 & 0
\end{array}\right]
$$




$$
\begin{aligned}
& \mathcal{I}:=\left\{\left(\eta^{\left\lceil\frac{k}{2}\right\rceil}\left(v_{\theta-1}^{b}\right)\right)^{T}\left(F_{b}+H_{b}\right) \eta^{\left\lceil\frac{k}{2}\right\rceil}\left(v_{\theta-1}^{b}\right)+\int_{\Omega}\left[\left(\eta^{\left\lceil\frac{k}{2}\right\rceil}\left(v_{\theta}(u)\right)\right)^{T} F_{i}(x) \eta^{\left\lceil\frac{k}{2}\right\rceil}\left(v_{\theta}(u)\right)+\frac{\mathrm{d}}{\mathrm{d} x}\left(\left(\eta^{\left\lceil\frac{k}{2}\right\rceil}\left(v_{\theta-1}(u)\right)\right)\right)^{T} H_{i}(x) \eta^{\left\lceil\frac{k}{2}\right\rceil}\left(v_{\theta-1}(u)\right)\right)\right] \mathrm{d} x \\
& \left.\mid F_{b} \in \mathcal{F}_{b}(k, \theta), F_{i} \in \mathcal{F}_{i}(k, \theta),\left(\eta^{\left\lceil\frac{k}{2}\right\rceil}\left(v_{\theta-1}^{b}\right)\right)^{T} H_{b} \eta^{\left\lceil\frac{k}{2}\right\rceil}\left(v_{\theta-1}^{b}\right)+\left[\left(\eta^{\left\lceil\frac{k}{2}\right\rceil}\left(v_{\theta-1}(u(x))\right)\right)^{T}\left(H_{i}(x)\right) \eta^{\left\lceil\frac{k}{2}\right\rceil}\left(v_{\theta-1}(u(x))\right)\right]_{x=0}^{1}=0\right\} \\
& \frac{\mathrm{d}}{\mathrm{d} x}\left(\left(v_{\theta-1}(u)\right)^{T} H_{i}(x)\left(v_{\theta-1}(u)\right)\right)=\left(v_{\theta}\right)^{T}\left[\begin{array}{ccc}
\frac{\mathrm{d}}{\mathrm{d} x} h_{11}(x) & \frac{\mathrm{d}}{\mathrm{d} x} h_{12}(x)+h_{11}(x) & \frac{1}{2} h_{12}(x) \\
\frac{\mathrm{d} x}{\mathrm{~d} x} h_{12}(x)+h_{11}(x) & \frac{\mathrm{d}}{\mathrm{d} x} h_{22}(x)+h_{12}(x) & h_{22}(x) \\
\frac{1}{2} h_{12}(x) & h_{22}(x) & 0
\end{array}\right]\left(v_{\theta}\right)
\end{aligned}
$$

and the terms characterising the multiplicity of the integral as described by (7) are obtained with (8) and with

$$
\left(v_{\theta-1}^{b}\right)^{T} H_{b}\left(v_{\theta-1}^{b}\right)=\left(v_{\theta-1}^{b}\right)^{T}\left[\begin{array}{cc}
-H_{i}(1) & 0 \\
0 & H_{i}(0)
\end{array}\right]\left(v_{\theta-1}^{b}\right) .
$$

Remark 1. Further to the non-uniqueness associated to the algebraic relations in the vector describing the quadratic representation, which is characterised by the sets $\mathcal{F}_{b}, \mathcal{F}_{i}$, the Fundamental Theorem of Calculus exposes the nonuniqueness of the integral expression associated to the differential relations of the elements in $v_{\theta}(u)$, characterising the set (7).

In order to simplify the presentation of the next result, let us introduce the function $\bar{H}_{i}(x)$, which satisfies

$$
\begin{aligned}
\frac{\mathrm{d}}{\mathrm{d} x}\left(\left(\eta^{\left\lceil\frac{k}{2}\right\rceil}\left(v_{\theta-1}(u)\right)\right)^{T} H_{i}(x) \eta^{\left\lceil\frac{k}{2}\right\rceil}\left(v_{\theta-1}(u)\right)\right) \\
=\left(\eta^{\left\lceil\frac{k}{2}\right\rceil}\left(v_{\theta}(u)\right)\right)^{T} \bar{H}_{i}(x) \eta^{\left\lceil\frac{k}{2}\right\rceil}\left(v_{\theta}(u)\right)
\end{aligned}
$$

and allows us to denote the quadratic form in the integrand of (7) in terms of matrix $F_{i}(x)+\bar{H}_{i}(x)$. The quadraticlike characterization of the integrand in terms of the algebraic and the differential relations leads to conditions for integral inequalities in terms of matrix inequalities as follows:

Theorem 1. If there exist $F_{b} \in \mathcal{F}_{b}, F_{i}(x) \in \mathcal{F}_{i}$, satisfying (1)-(2), and $H_{i}(x) \in \mathcal{C}^{1}(\Omega)$, yielding $H_{b}$ as in (7) and $\bar{H}_{i}(x)$ as in (9) such that

$$
\begin{gathered}
\left(\eta^{\bar{k}}\left(v_{\theta-1}^{b}\right)\right)^{T}\left(F_{b}+H_{b}\right) \eta^{\bar{k}}\left(v_{\theta-1}^{b}\right) \geq 0 \quad \forall u \in \mathcal{B}(B), \\
F_{i}(x)+\bar{H}_{i}(x) \geq 0 \quad \forall x \in \Omega,
\end{gathered}
$$

where $\bar{k}=\left\lceil\frac{k}{2}\right\rceil$, then inequality (4) holds in the subspace defined by $\mathcal{B}(B)$.

Proof: For given polynomials $f_{b}$ and $f_{i}$ satisfying $k=$ $\max \left(\operatorname{deg}\left(f_{b}\right), \operatorname{deg}\left(f_{i}\right)\right)$ we can express an integral expresion as in (4), say $\phi(u)$, using the quadratic forms as defined in (1)-(2) with $F_{b} \in \mathcal{F}_{b}(k, \theta), F_{i}(x) \in \mathcal{F}_{i}(k, \theta)$, which gives

$$
\begin{aligned}
\phi(u)= & f_{b}\left(v_{\theta-1}^{b}\right)+\int_{\Omega} f_{i}\left(x, v_{\theta}(u)\right) \mathrm{d} x, \\
= & \left(\eta^{\bar{k}}\left(v_{\theta-1}^{b}\right)\right)^{T} F_{b} \eta^{\bar{k}}\left(v_{\theta-1}^{b}\right) \\
& +\int_{\Omega}\left(\eta^{\bar{k}}\left(v_{\theta}(u)\right)\right)^{T} F_{i}(x) \eta^{\bar{k}}\left(v_{\theta}(u)\right) \mathrm{d} x .
\end{aligned}
$$

Following the definition of set $\mathcal{I}$ in $(7)$ and the definition of $\bar{H}_{i}$ in (9) we obtain

$$
\begin{aligned}
& \phi(u)=\left(\eta^{\bar{k}}\left(v_{\theta-1}^{b}\right)\right)^{T}\left(F_{b}+H_{b}\right) \eta^{\bar{k}}\left(v_{\theta-1}^{b}\right) \\
& \quad+\int_{\Omega}\left(\eta^{\bar{k}}\left(v_{\theta}(u)\right)\right)^{T}\left(F_{i}(x)+\bar{H}_{i}(x)\right) \eta^{\bar{k}}\left(v_{\theta}(u)\right) \mathrm{d} x .
\end{aligned}
$$

Hence, if the boundary term satisfies (10), and the integral term satisfies (11) then $\phi(u) \geq 0 \forall u \in \mathcal{B}(B)$.

Remark 2. Inequality (11) is a differential matrix inequality since the elements $\bar{H}_{i}(x)$ involve continuously differentiable functions and their derivatives.

\section{A. Semidefinite programming formulation}

Whenever matrix $F_{i}(x)$ is a polynomial on variable $x$ and we impose polynomial dependence of $\bar{H}_{i}(x)$ on variable $x$, inequality (11) can be addressed by a straightforward application of Putinar's Positivstellensatz [14, Theorem 2.14]. Note that the set $\Omega=[0,1]$, can be described as the semi-algebraic set $\{x \mid \omega(x):=x(1-x) \geq 0\}$.

Corollary 1. For $F_{i}(x)+\bar{H}_{i}(x) \in \mathcal{R}^{n_{d} \times n_{d}}[x]$, if there exists $N(x) \in \Sigma^{n_{M} \times n_{M}}[x]$ such that

$$
F_{i}(x)+\bar{H}_{i}(x)-N(x) \omega(x) \in \Sigma^{n_{M} \times n_{M}}[x]
$$

then (11) holds.

Remark 3. If the coefficients of $F_{i}(x)$ and $\bar{H}_{i}(x)$ depend affinely in unknown parameters and the degree of $N(x)$ is fixed, checking whether (13) holds can be cast as a feasibility test of a convex set of constraints, an SDP, whose dimension depends on the degree of $F_{i}(x)+\bar{H}_{i}(x)$ and $N(x)$ and on the dimension of matrix $F_{i}(x)+\bar{H}_{i}(x)$ which depends on the degree $k$ and the order $\theta$ as in (1)-(2).

The formulation of inequalities (10) and (11) is possible thanks to the application of the Fundamental Theorem of Calculus to characterize the set of quadratic-like representations of an integral inequality, as described by the set (7). The terms introduced in the integrand by matrix $H_{i}$ do not affect the value of the integral and allow for a test for positivity based on the positivity of the matrices in the quadratic-like representation. This is reminiscent of the quadratic representation that is used in sum-ofsquares when checking positivity of a polynomial. Also, for polynomial expressions, the algebraic relations in the quadratic representation of integrand polynomials are here defined in terms of functions, (see (6)) instead of scalars. With the solution to (10) it is possible to verify inequalities in subspaces as in (3), incorporating boundary values of the dependent variables. 


\section{Stability Analysis for Partial Differential EQUATIONS}

This section recalls some results for the stability analysis of PDEs and formulates integral inequalities for the convergence of norms of the solutions of PDEs with polynomial dependence on the dependent variables. Results extending Lyapunov's second method to PDE systems can be found in [17], [20], [31] and [12]. The formulation detailed in the latter is recalled below:

Definition 1. A nonlinear semi-group on a compact normed space $C$ is a family of maps $\left\{S(t) \mid C \rightarrow C, t \geq t_{0}\right\}$ such that

- for each $t \geq t_{0}, S(t)$ is continuous from $C$ to $C$,

- for each $u \in C$, the mapping $t \rightarrow S(t) u$ is continuous,

- $S(0)$ is the identity on $C$,

- $S(t)(S(\tau) u)=S(t+\tau) u$ for all $u \in C$ and all $t, \tau \geq 0$.

Definition 2. Let $\left\{S(t), t \geq t_{0}\right\}$ be a nonlinear semigroup on $C$ and for any $u \in C$, let $Y(u)=\left\{S(t) u, t \geq t_{0}\right\}$ be the orbit through $u$. We say $u$ is an equilibrium point if $Y(u)=\{u\}$

An orbit $Y(u)$ is stable if for any $\epsilon>0$, there exists $\delta(\epsilon)>0$ such that for all $t \geq t_{0},\|S(t) u-S(t) v\|_{C}<\epsilon$ whenever $\|u-v\|_{C}<\delta(\epsilon), v \in C$, where $\|\cdot\|_{C}$ is the norm defined on $C$. An orbit is uniformly asymptotically stable if it is stable and also there is a neighbourhood $D=\{v \in C \mid$ $\left.\|u-v\|_{C}<r\right\}$ such that $\|S(t) u-S(t) v\|_{C} \rightarrow 0$ as $t \rightarrow \infty$, uniformly for $v \in D^{1}$. Similarly, it is exponentially stable if there exist $\nu, \gamma>0$ such that

$$
\|S(t) u-S(t) v\|_{C} \leq \gamma\|u-v\|_{C} e^{-\nu\left(t-t_{0}\right)},
$$

for all $t \geq t_{0}$ and all $u, v \in C$.

Definition 3. Let $\left\{S(t), t \geq t_{0}\right\}$ be a nonlinear semigroup on $C$. A Lyapunov function is a continuous realvalued function $V$ on $C$ such that

$$
\frac{d}{d t} V(u)=\lim _{t \rightarrow 0^{+}} \frac{V(S(t) u)-V(u)}{t} \leq 0,
$$

for all $u \in C$.

Theorem 2. (Lyapunov Theorem for Nonlinear Semigroups, [12, Theorem 4.1.4]), Let $\left\{S(t), t \geq t_{0}\right\}$ be a nonlinear semi-group, and let 0 be an equilibrium point in $C$. Suppose $V$ is a Lyapunov function on $C$ which satisfies $V(0)=0$, and $V(u) \geq \alpha_{1}\|u\|_{C}$ for $\alpha_{1}>0$ and $u \in C$. Then, 0 is stable. In addition, if $\partial_{t} V(u) \leq-\alpha_{2}\|u\|_{C}$ for $\alpha_{2}>0$, then 0 is uniformly asymptotically stable.

For the proof of the above theorem, refer to [12, p.84]. In [5, Theorem 5.1.3], necessary and sufficient conditions for a linear operator defining a PDE to be the infinitesimal generator of exponentially stable strongly continuous semigroups on a Hilbert space is given in terms of the existence of a positive operator satisfying a Lyapunov equation. Such a theorem is based on the results in [6]. The extension of this result for more general spaces has

$$
{ }^{1} \text { i.e. } \forall \varepsilon>0, \exists T>0: t>T \text { s.t. }\|S(t) u-S(t) v\|_{C}<\varepsilon, \forall v \in D .
$$

been provided in [22]. The above Theorem establishes conditions for the stability and uniform asymptotic stability of nonlinear semigroups. Please refer to [16] for conditions on nonlinear operators to generate semigroups of nonlinear contractions. The remaining of this section proposes a sufficient condition for the existence of Lyapunov function for PDEs defined by nonlinear polynomial operators. In what follows, we present the class of PDE systems and Lyapunov functionals studied in this paper. Consider the following PDE system

$$
\partial_{t} u=F\left(x, D^{\alpha} u\right), \quad u\left(t_{0}, x\right)=u_{0}(x) \in \mathcal{M} \subset \mathcal{H}^{q}(\Omega)
$$

where $q \in \mathbb{N}_{0}$. Let $F\left(x, D^{\alpha} u\right)=\mathcal{N} u$, where $\mathcal{N}$ is a nonlinear (polynomial) operator defined on $\mathcal{M}$, a closed subset of $\mathcal{H}^{q}(\Omega)$. Continuous solutions to the PDE (15) exist in $\mathcal{M}$ and are unique, provided $\mathcal{N}$ generates a nonlinear semigroup of contractions.

The following theorem is a Lyapunov result for the exponential convergence of the $\mathcal{H}^{q}$ norm of the solutions to $(15)$.

Theorem 3. Suppose there exist a functional $V \in \mathcal{C}^{1}$, with $V(0)=0$, and scalars $c_{1}, c_{2}, c_{3} \in \mathbb{R}_{>0}$ such that

$$
\begin{gathered}
c_{1}\|u\|_{q}^{2} \leq V(u) \leq c_{2}\|u\|_{q}^{2} \\
\frac{d V(u)}{d t} \leq-c_{3}\|u\|_{q}^{2}
\end{gathered}
$$

then, the $\mathcal{H}^{q}$ norm of the trajectories of (15) satisfy

$$
\|u(t, x)\|_{q}^{2} \leq \frac{c_{2}}{c_{1}}\left\|u_{0}(x)\right\|_{q}^{2} e^{-\frac{c_{3}}{c_{1}}\left(t-t_{0}\right)}
$$

where $u_{0}=u\left(t_{0}, x\right)$.

Proof: From (16), (17), one has $\frac{\frac{\mathrm{d} V(u)}{\mathrm{d} t}}{V(u)} \leq-\frac{c_{3}}{c_{1}}$.

Since $\frac{\frac{\mathrm{d} V(u)}{\mathrm{d} t}}{V(u)}=\frac{\mathrm{d}(\ln (V(u)))}{\mathrm{d} t}$, the integral in time of the above expression over $\left[t_{0}, t\right]$ yields $\ln (V(u(t)))-$ $\ln \left(V\left(u\left(t_{0}\right)\right)\right) \leq-\frac{c_{3}}{c_{1}}\left(t-t_{0}\right)$ which gives $V(u(t)) \leq$ $V\left(u\left(t_{0}\right)\right) e^{-\frac{c_{3}}{c_{1}}\left(t-t_{0}\right)}$. Finally (18) is obtained by applying the bounds of (16) on the above inequality.

Consider candidate Lyapunov functionals of the form

$$
V(u)=\frac{1}{2} \int_{\Omega}\left(D^{q} u\right)^{T} P(x)\left(D^{q} u\right) \mathrm{d} x, P(x)>0 \forall x \in \Omega .
$$

That is, $V(u)=\frac{1}{2}\|u\|_{(q, P(x))}^{2}$, the squared $P(x)$-weighted $\mathcal{H}^{q}$-norm. The following lemma states the equivalence of the weighted norm and the $\mathcal{H}^{q}$-norm.

Lemma 1. If $P(x)>0 \quad \forall x \in \Omega$, then the norms $\|u\|_{(q, P)}$ and $\|u\|_{q}$ are equivalent.

The proof consists of showing that the inequalities $\sqrt{\lambda_{m}}\|u\|_{q} \leq\|u\|_{(q, P)} \leq \sqrt{\lambda_{M}}\|u\|_{q}$ hold, with $\lambda_{m}>0$, $\lambda_{M}>0$ satisfying $\lambda_{m} I \leq P(x) \leq \lambda_{M} I, \forall x \in \Omega$.

Remark 4. For $q_{1}<q_{2}$, the space $\mathcal{H}^{q_{1}}$ is embedded in $\mathcal{H}^{q_{2}}$ [8, Sec 5.6]. Therefore, stability in $\mathcal{H}^{q_{2}}$-norm implies stability in $\mathcal{H}^{q_{1}}$-norm, but the converse does not necessarily hold. 
Proposition 1. If there exists a function $P(x)$ and positive scalars $\epsilon_{1}, \epsilon_{2}$ such that

$$
\begin{array}{r}
\int_{\Omega}\left[\left(D^{q} u\right)^{T} P(x)\left(D^{q} u\right)-\epsilon_{1}\left(D^{q} u\right)^{T}\left(D^{q} u\right)\right] \mathrm{d} x \geq 0 \quad(20) \\
-\int_{\Omega}\left[\left(D^{q} u\right)^{T} P(x) F\left(x, D^{\alpha} u\right)+F^{T}\left(x, D^{q} u\right) P(x)\left(D^{q} u\right)\right. \\
\left.+\epsilon_{2}\left(D^{q} u\right)^{T}\left(D^{q} u\right)\right] \mathrm{d} x \geq 0 \quad(21)
\end{array}
$$

then the $\mathcal{H}^{q}$ norm of solutions to (15) satisfy (18) with $c_{1}=\min _{\Omega}\left(\lambda_{\min }(P(x))\right), c_{2}=\max _{\Omega}\left(\lambda_{\max }(P(x))\right)$, and $c_{3}=\epsilon_{2}$.

The verification of inequalities (20), (21) is a sufficient condition to verify the conditions of Theorem 3 , since it restricts the class of functions in (16), (17) to be as in (19).

Inequalities (20), (21) are integral inequalities such as the ones studied in Section II. The sets $\mathcal{B}(B)$ as in (3) associated to the inequalities are defined by the domain of the PDE operators. The results of Section II-A can therefore be applied to (20)-(21) whenever the integrands are polynomial functions. In this context, $P(x)$ is a variable parameterized in terms of polynomial coefficients. For such a parameterization a tradeoff must be accounted for: as the class of parameterized functions is increased by adding elements elements of the basis (increasing the degree of a polynomial $P(x)$ )the computational burden required to solve (13) increases.

\section{EXAMPLES}

In this section we present solutions to inequalities (20), (21) by solving the SOS constraints obtained with the formulation of Corollary 1, (the constraints are cast as SDP with SOSTOOLS [18] and solved with SeDuMi [26]). The exponential stability of the $\mathcal{L}_{2}$ norm of the solution of a simple hyperbolic equation is studied below. Example A) Consider the equation

$$
\partial_{t} u=-\partial_{x} u \quad x \in[0,1], t>0 \quad u(t, 0)=0,
$$

which gives $\mathcal{B}=\left\{u \in \mathcal{C}^{2}(\Omega) \mid\left[\begin{array}{ll}0 & 1\end{array}\right]\left[\begin{array}{l}u(1) \\ u(0)\end{array}\right]=0\right\}$. Given $\lambda>0$, let $E_{p}=\frac{1}{2} \int_{\Omega} p(x) u^{2}(x) \mathrm{d} x$, be the candidate function to certify $-\lambda E_{p}-\dot{E}_{p}=\int_{\Omega}-\frac{1}{2} \lambda p(x) u^{2}(x)+$ $p(x) u \partial_{x} u(x) \mathrm{d} x \geq 0$ (proving the exponential stability with convergence rate $\lambda$ ). We apply Theorem 1 to the Lyapunov inequalities in Proposition 1 to obtain

$$
\begin{gathered}
p(x)>0 \forall x \in \Omega \\
{\left[\begin{array}{l}
u(1) \\
u(0)
\end{array}\right]^{T}\left[\begin{array}{cc}
-h(1) & 0 \\
0 & h(0)
\end{array}\right]\left[\begin{array}{l}
u(1) \\
u(0)
\end{array}\right]>0 \forall\left[\begin{array}{l}
u(1) \\
u(0)
\end{array}\right] \in \mathcal{B}} \\
(23 \mathrm{~b}) \\
M(x)=\frac{1}{2}\left[\begin{array}{cc}
-\lambda p(x)-\frac{d}{d x} h(x) & (h(x)-p(x)) \\
(h(x)-p(x)) & 0
\end{array}\right] \geq 0 \\
\quad \forall x \in \Omega . \quad(23 \mathrm{c})
\end{gathered}
$$

Solve (23c) by imposing $h(x)=-p(x)$ and by solving the differential equation $\partial_{x} h(x)+\lambda h(x)=0$, to obtain

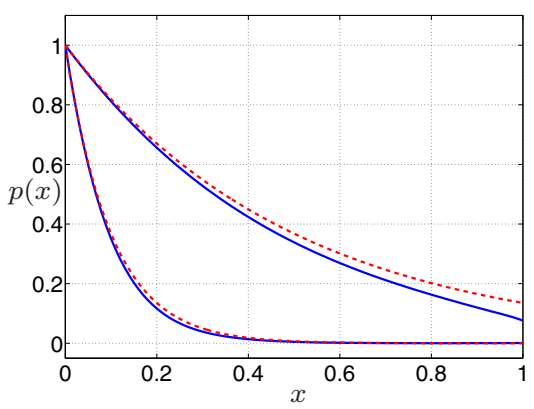

Fig. 1: Weighting functions proving exponential stability for convergence rates $\lambda \in\{2,10\}$. The red dotted curves depict the solution $p(x)=e^{-\lambda x}$. The solid blue lines correspond to the polynomials obtained by solving (24).

$h(x)=-e^{-\lambda x}$, which satisfies (23a) (i.e. $-h(x)=p(x)>$ $0)$. Notice that with $p(x)=-h(x)=e^{-\lambda x}$ the inequality of (23c) holds for all $x \in \mathbb{R}$. Inequality (23b) is expressed as $-h(1) u^{2}(1)>0$, which clearly holds since $-h(1)=e^{-\lambda}>$ 0 . The inequalities then hold for any $\lambda>0$ which proves the exponential stability of the $\mathcal{L}_{2}$ norm of the solution for any convergence rate. This is expected as, for bounded domains, the equation presents finite-time stability.

The inequalities in (20)-(21) were also formulated with polynomial weighting function $p(x)$, with $q=0$ (giving $\left.D^{q} u=u\right)$ and $\epsilon_{2}=\lambda$. Theorem 1 is applied to the resulting inequalities and we use the Positivstellensatz to formulate the SOSP as in Corollary 1

Find $p, h, N \quad$ subject to $\begin{aligned} & M(x)+N(x) x(x-1) \in \Sigma^{2 \times 2}[x], \\ & N(x) \in \Sigma^{2 \times 2}[x] .\end{aligned}$

Solutions to the above inequalities were obtained for $\lambda \in$ $(0,10]$ (the value $\lambda^{*}=10$ was solved with $\operatorname{deg}(p(x))=$ $\operatorname{deg}(h(x))=30)$. The numerical results provide polynomial Lyapunov certificates for the $\mathcal{L}_{2}$ stability of the solutions of (22). A comparison of the solution $p(x)=e^{-\lambda \theta}$ and the numerical solutions are depicted in Figure 1.

In the next example we study the stability of a nonlinear, inhomogeneous PDE.

Example B) (System of nonlinear inhomogeneous PDEs) Consider the following PDE

$$
\partial_{t} u=R^{-1} \nabla^{2} u+C(x) u+u \times(D(x) \nabla u)
$$

where $u(\cdot, x): \mathbb{R} \rightarrow \mathbb{R}^{3}, R>0$ and

$$
C(x)=\left[\begin{array}{ccc}
0 & 0 & 0 \\
x & 0 & 0 \\
x & -x^{2} & 0
\end{array}\right] ; D(x)=\left[\begin{array}{ccc}
0 & 0 & 0 \\
0 & 0 & 0 \\
0 & 0 & \left(-\frac{1}{2}+x\right)
\end{array}\right]
$$

subject to the boundary conditions $u(0)=u(1)=0$. In order to obtain the asymptotic stability bound in terms of the parameter $R$ a straightforward approach is to take energy as the Lyapunov function. In this case, the nonlinear terms, given by $u \times(D(x) \nabla u)=$ $\left(-\frac{1}{2}+x\right)\left[u_{2} \partial_{x} u_{3}-u_{1} \partial_{x} u_{3} 0\right]^{T}$, are removed from the expression of the time-derivative of the energy and the 
remaining expression is influenced only by the linear terms $R^{-1} \nabla^{2} u+C(x) u$. This choice results in conservatism since the stability may not be certified by the energy of the state taken as the Lyapunov function. Instead consider a weighted $\mathcal{L}_{2}$ energy as a LF candidate and solve (20), (21) to obtain $P(x)$, the weighting function. In order to illustrate this solution, we compute the largest value of parameter $R$ for which stability could be certified both with energy and the weighted $\mathcal{L}_{2}$ norm.

The results are depicted in Table I.

TABLE I: Stability intervals for parameter $R \in\left(0, R^{*}\right]$ for different degrees of $P(x)$.

\begin{tabular}{|c||c|c|c|c|}
\hline $\operatorname{deg}(P(x))$ & $0, P(x)=I$ & 1 & 2 & 3 \\
\hline$R^{*}$ & 6.3 & 10.5 & 17.5 & 21 \\
\hline
\end{tabular}

\section{CONCLUSion}

This paper proposed tests for positivity of integral expressions with integrands that are polynomial on the dependent variables. A set of quadratic-like representations for the polynomial integrand is obtained with the Fundamental Theorem of Calculus. This way, the value of the dependent variables at the boundary of the integration domain can be accounted for. The positivity of the integral is then studied by analyzing the matrices associated to the quadratic-like representation. Under the assumption that the integrand is also polynomial on the independent variable, matrix positivity tests are cast as SDPs. We then studied integral inequalities from Lyapunov stability conditions for PDEs. The proposed Lyapunov functionals are given by weighted $\mathcal{H}^{q}$-norms. Two examples were presented to illustrate the effectiveness of the proposed approach. Future work will cover the problems of synthesis of in-domain and boundary controllers, [13], [9] and the extension to integrals over domains of two spatial dimensions, of which [28] reports some preliminary results.

\section{REFERENCES}

[1] F. Bribiesca Argomedo, C. Prieur, E. Witrant, and S. Bremond, "A strict control Lyapunov function for a diffusion equation with time-varying distributed coefficients," Automatic Control, IEEE Transactions on, vol. 58, no. 2, pp. 290-303, 2013.

2] F. Castillo, E. Witrant, C. Prieur, and L. Dugard, "Boundary observers for linear and quasi-linear hyperbolic systems with application to flow control," Automatica, vol. 49, no. 11, pp. $3180-3188,2013$

[3] M. Choi, T. Lam, and B. Reznick, "Sums of squares of real polynomials," in Symposia in Pure Mathematics, vol. 58, no. 2, 1995, pp. 103-126.

[4] J.-M. Coron and B. D'Andrea-Novel, "Stabilization of a rotating body beam without damping," Automatic Control, IEEE Transactions on, vol. 43, no. 5, pp. 608-618, May 1998.

[5] R. F. Curtain and H. J. Zwart, An Introduction to InfiniteDimensional Linear Systems Theory, ser. Texts in Applied Mathematics. Berlin: Springer-Verlag, 1995, vol. 21.

[6] R. Datko, "Extending a theorem of A. M. Liapunov to Hilbert space," Journal of Mathematical Analysis and Applications, vol. 32 , no. 3 , pp. $610-616,1970$.

[7] N. H. El-Farra, A. Armaou, and P. D. Christofides, "Analysis and control of parabolic PDE systems with input constraints," Automatica, vol. 39, no. 4, pp. 715 - 725, 2003.
[8] L. C. Evans, Partial Differential Equations, 2nd ed., ser. Graduate Studies in Mathematics. American Mathematical Society, 2010.

[9] A. Gahlawat and M. Peet, "Designing observer-based controllers for PDE systems: A heat-conducting rod with point observation and boundary control," in Decision and Control and European Control Conference (CDC-ECC), 2011 50th IEEE Conference on, Dec 2011, pp. 6985-6990.

[10] P. J. Goulart and S. Chernyshenko, "Global stability analysis of fluid flows using sum-of-squares," Physica D: Nonlinear Phenomena, vol. 241, no. 6, pp. $692-704,2012$.

[11] D. Henrion and M. Korda, "Convex computation of the region of attraction of polynomial control systems," Automatic Control, IEEE Transactions on, vol. 59, no. 2, pp. 297-312, Feb 2014.

[12] D. Henry, Geometric Theory of Semilinear Parabolic Equations, ser. Lecture Notes in Mathematics. Berlin: Springer-Verlag, 1981.

[13] M. Krstić and A. Smyshlyaev, Boundary control of PDEs : a course on backstepping designs, ser. Advances in design and control. Philadelphia, PA: Society for Industrial and Applied Mathematics, 2008.

[14] J. B. Lasserre, Moments, Positive Polynomials and Their Applications. Imperial College Press, London, 2009.

[15] J. B. Lasserre, D. Henrion, C. Prieur, and E. Trelat, "Nonlinear optimal control via occupation measures and LMI relaxations." SIAM J. Control and Optimization, vol. 47, no. 4, pp. 1643$1666,2008$.

[16] I. Miyadera, Nonlinear Semigroups. American Mathematical Society, 1993.

[17] A. Movchan, "The direct method of Liapunov in stability problems of elastic systems," Journal of Applied Mathematics and Mechanics, vol. 23, no. 3, pp. 686-700, 1959.

[18] A. Papachristodoulou, J. Anderson, G. Valmorbida, S. Prajna, P. Seiler, and P. Parrilo, "SOSTOOLS: Sum of squares optimization toolbox for MATLAB V3.00," 2013.

[19] A. Papachristodoulou and M. Peet, "On the analysis of systems described by classes of partial differential equations," in Decision and Control, 2006 45th IEEE Conference on, 2006, pp. 747-752.

[20] P. C. Parks and A. J. Pritchard, "On the construction and use of Lyapunov functionals," in 4th IFAC World Congress, 1969, pp. 59-76.

[21] P. Parrilo, "Structured semidefinite programs and semialgebraic geometry methods in robustness and optimization," Ph.D. dissertation, California Institute of Technology, 2000.

[22] A. Pazy, Semigroups of Linear Operators and Applications to Partial Differential Equations, 1st ed., ser. Applied Mathematical Sciences. Springer-Verlag New York, 1983, vol. 44.

[23] M. M. Peet, A. Papachristodoulou, and S. Lall, "Positive forms and stability of linear time-delay systems," SIAM J. Control and Optimization, vol. 47, no. 6, pp. 3237-3258, 2007.

[24] S. Prajna, A. Papachristodoulou, and F. Wu, "Nonlinear control synthesis by sum of squares optimization: a Lyapunov-based approach," in 5'th Asian Control Conference, vol. 1, 2004, pp. $157-165$.

[25] B. Straughan, The Energy Method, Stability, and Nonlinear Convection, 2nd ed., ser. Applied Mathematical Sciences. Berlin: Springer-Verlag, 2004, vol. 91.

[26] J. F. Sturm, "Using SeDuMi 1.02, a MATLAB toolbox for optimization over symmetric cones," 1998.

[27] G. Valmorbida, M. Ahmadi, and A. Papachristodoulou, "Semidefinite programming and functional inequalities for distributed parameter systems," in Decision and Control, 2014 53rd IEEE Conference on, Dec 2014, pp. 4304-4309.

[28] - "Convex solutions to integral inequalities in twodimensional domains," in Decision and Control, 2015 54th IEEE Conference on, Dec 2015, pp. -.

[29] G. Valmorbida, S. Tarbouriech, and G. Garcia, "Design of polynomial control laws for polynomial systems subject to actuator saturation," Automatic Control, IEEE Transactions on, vol. 58, no. 7, pp. 1758-1770, July 2013.

[30] R. Vazquez, S. E., and M. Krstic, "Magnetohydrodynamic state estimation with boundary sensors," Automatica, vol. 44, no. 10, pp. $2517-2527,2008$.

[31] A. J. Walker, Dynamical Systems and Evolution Equations: Theory and applications. New York: Plenum Press, 1980. 